Nico Lüdtke $\cdot$ Hironori Matsuzaki (Hrsg.)

Akteur - Individuum - Subjekt 
Nico Lüdtke $\cdot$ Hironori Matsuzaki (Hrsg.)

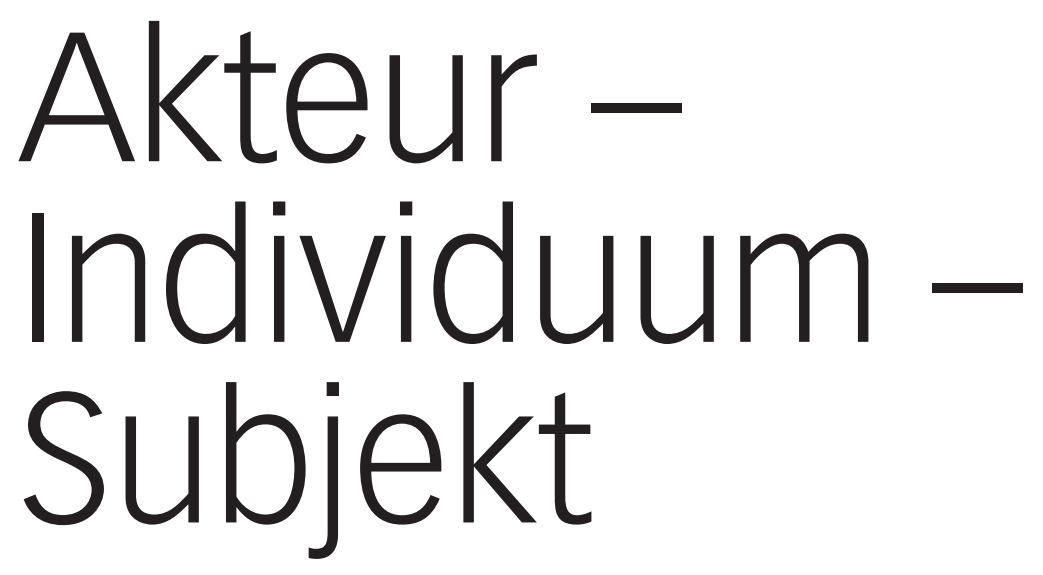

Fragen zu ,Personalität' und ,Sozialität' 
Bibliografische Information der Deutschen Nationalbibliothek

Die Deutsche Nationalbibliothek verzeichnet diese Publikation in der

Deutschen Nationalbibliografie; detaillierte bibliografische Daten sind im Internet über

$<$ http://dnb.d-nb.de> abrufbar.

\section{Auflage 2011}

Alle Rechte vorbehalten

๑) VS Verlag für Sozialwissenschaften | Springer Fachmedien Wiesbaden GmbH 2011

Lektorat: Katrin Emmerich

VS Verlag für Sozialwissenschaften ist eine Marke von Springer Fachmedien.

Springer Fachmedien ist Teil der Fachverlagsgruppe Springer Science+Business Media.

www.vs-verlag.de

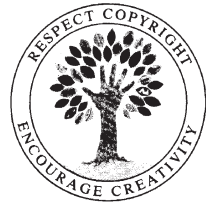

Das Werk einschließlich aller seiner Teile ist urheberrechtlich geschützt. Jede Verwertung außerhalb der engen Grenzen des Urheberrechtsgesetzes ist ohne Zustimmung des verlags unzulässig und strafbar. Das gilt insbesondere für Vervielfältigungen, Übersetzungen, Mikroverfilmungen und die Einspeicherung und Verarbeitung in elektronischen Systemen.

Die Wiedergabe von Gebrauchsnamen, Handelsnamen, Warenbezeichnungen usw. in diesem Werk berechtigt auch ohne besondere Kennzeichnung nicht zu der Annahme, dass solche Namen im Sinne der Warenzeichen- und Markenschutz-Gesetzgebung als frei zu betrachten wären und daher von jedermann benutzt werden dürften.

Umschlaggestaltung: KünkelLopka Medienentwicklung, Heidelberg Gedruckt auf säurefreiem und chlorfrei gebleichtem Papier Printed in Germany

ISBN 978-3-531-17854-7 


\section{Inhalt}

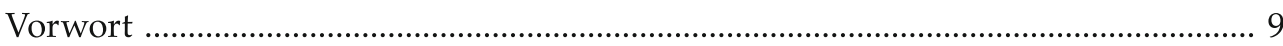

Nico Lüdtke

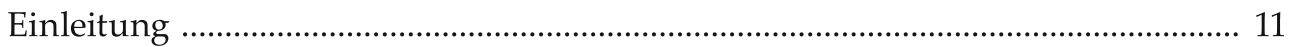

\section{Sozialtheoretische Positionen in der Diskussion}

Uwe Schimank

So viel zu Akteuren! Ein Minimalkonzept zur Beantwortung

einer Vorfrage soziologischer Erklärungen

Andrea Maurer

„Akteure“ in soziologischen Erklärungen

Martin Endreß

Individualität und Sozialität im Kontext verstehender Soziologie:

Max Weber und Alfred Schütz

Rainer Greshoff

Was sind die aktiv-dynamischen Kräfte der Produktion des Sozialen?

Wolfgang Ludwig Schneider

Akteure oder Personen/psychische Systeme?

Niklas Woermann

"The phenomenon exhibits its staff as a population" -

Die reflexive Akteurskonzeption der Ethnomethodologie 
Gregor Bongaerts

Vom Sichtbaren und Unsichtbaren sozialer Akteure Überlegungen zum Akteursbegriff im Rahmen von Bourdieus Theorie der Praxis

Wil Martens

Der Akteur: Habitus, Intention und Reflexion 171

Frank Meier

Die Akteure des soziologischen Neo-Institutionalismus 199

\section{Die aktuelle Kontroverse zum Verhältnis von Anthropologie und Sozialtheorie}

Jens Greve

Menschliche Aktorenschaft

Nico Lüdtke

Die konstitutiven Bedingungen von Personalität und Sozialität - Konzeptuelle Antworten von George Herbert Mead und Helmuth Plessner

Henning Laux

Latours Akteure. Ein Beitrag zur Neuvermessung der Handlungstheorie

Hironori Matsuzaki

Die Frage nach der "Agency" von Technik und die Normenvergessenheit der Techniksoziologie 301

\section{Sozialtheoretisch reflektierte Gesellschaftsanalyse - historische und normative Perspektiven}

Gesa Lindemann

Die Akteure der funktional differenzierten Gesellschaft 
Gregor Fitzi

Der gesellschaftliche Aufbau der Person

Simmels Beitrag zu einer soziologischen Anthropologie

351

Anna Henkel

Freigestellte Person. Soziologische Reflektion

einer marginalisierten Zurechnungsadresse 369

Sigrid Graumann

Anerkennung und Sorgebeziehungen

Personenregister 401

Autorinnen und Autoren 409 


\section{Vorwort}

Im Januar 2010 fand an der Carl von Ossietzky Universität Oldenburg eine Arbeitstagung mit dem Titel „Akteur - Individuum - Subjekt: Fragen zu ,Personalität' und ,Sozialität"“ statt, organisiert von Nico Lüdtke, Hironori Matsuzaki, Rainer Greshoff und Gesa Lindemann. Die Resonanz war überaus positiv. Die Veranstaltung zeigte, dass die Initiierung einer allgemeinen Diskussion um Akteur, Individuum, Subjekt und der damit zusammenhängenden Probleme von Personalität und Sozialität nicht nur von etablierten FachvertreterInnen begrüßt wird, sondern gerade auch unter jüngeren SozialwissenschaftlerInnen auf ein breites Interesse stößt. Die Beiträge stellten aktuelle Entwicklungen der sozialtheoretischen Diskussion dar und machten insgesamt deutlich, dass das Themenfeld derzeit besondere Aufmerksamkeit genießt. Im Vergleich zur Tagung weist die Konzeption des Tagungsbandes eine Reihe von Erweiterungen auf. Um dem gegenwärtigen Forschungsspektrum möglichst Rechnung zu tragen, wurden neben den ausgearbeiteten Vorträgen auch Texte thematisch einschlägiger AutorInnen zu wichtigen Theoriepositionen aufgenommen, die auf der Tagung zunächst nicht vertreten waren. In diesem Sinne ist das anvisierte Ziel des Bandes nicht nur eine systematische Selbstvergewisserung der sozialtheoretischen Grundlagen einzelner Konzepte, sondern darüber hinaus ein Überblick über den Stand der Diskussion innerhalb der Disziplin. Außerdem soll der Dialog zwischen den Generationen weiter befördert werden. Bei der Auswahl der Beiträge stand deswegen das Bemühen im Vordergrund, ein ausgewogenes Verhältnis zwischen renommierten FachvertreterInnen und jüngeren WissenschaftlerInnen zu finden. Die Herausgeber hoffen, diesen Ansprüchen mit der vorliegenden Publikation gerecht geworden $\mathrm{zu}$ sein.

In erster Linie möchten wir den AutorInnen für die Zusammenarbeit und ihre guten Beiträge danken, die diesen Band möglich gemacht haben. Darüber hinaus sei Gesa Lindemann und Rainer Greshoff herzlich gedankt, die die Arbeit der Herausgeber stets unterstützt haben. Die reibungslose Fertigstellung des Bandes schließlich verdankt sich der entgegenkommenden Bereitschaft des VS Verlages und der freundlichen Betreuung Frank Engelhardts sowie Katrin Emmerichs.

Mai 2011

Oldenburg/Tokyo

Nico Lüdtke, Hironori Matsuzaki 